\title{
Violência suicida entre os gêneros na região metropolitana de Maceió/Alagoas, Brasil: 20 anos de análise
}

\author{
Suicidal violence between genders in the metropolitan region of Maceió/Alagoas, Brazil: \\ 20 years of analysis
}

Violencia suicida entre géneros en la región metropolitana de Maceió/Alagoas, Brasil: 20 años de análisis

Alessandra Vieira da Silva ${ }^{1 *}$, Géssyca Luyse Procópio Gonzaga ${ }^{1}$, Lívia Acioli Murta Torres ${ }^{1}$, Lucimara Rocha da Silva ${ }^{1}$, Gabrielly Maria Argolo Acioly ${ }^{1}$, Isabela Cristina Chaves Valente Reis ${ }^{1}$, Mara Cristina Ribeiro', Evanisa Helena Maio de Brum¹, Kevan Guilherme Nóbrega Barbosa1.

\section{RESUMO}

Objetivo: Realizar uma análise epidemiológica acerca do suicídio, dentre os gêneros, na região metropolitana de Maceió, ao longo de 20 anos (2000 até 2019). Métodos: Estudo ecológico observacional e transversal, realizado com amostra de 693 vítimas de suicídio residentes na região metropolitana de Maceió, entre ambos os gêneros. Foi utilizado como fonte de informação os dados de Sistema de Informação de Mortalidade (SIM) do Ministério da Saúde, disponibilizados pelas plataformas do DATASUS. Os dados foram analisados pela estatística temporal e espacial descritiva e pela análise inferencial de tendência de Mann-Kendall, adotando nível de significância de 5\%. Resultados: Do total de suicídios, $77,5 \%$ (537) eram do gênero masculino e $22,5 \%$ (155) eram mulheres. A taxa média para a região metropolitana foi de 2,2 por 100.000 habitantes, sendo 3,3 por 100.000 para homens e 1,0 por 100.000 para mulheres. Houve incremento significativo das taxas entre os anos de 2000 e 2009 para a população em geral $(p=0,03)$ e para o gênero feminino $(p=$ $0,01)$. Conclusão: Os homens foram as principais vítimas de suicídio com taxas mais altas e estáveis, já para o sexo feminino enquanto que houve incremento significativo das taxas para o sexo feminino entre 2000 e 2009.

Palavras-chave: Estudos de série temporal, Suicídio, Violência.

\section{ABSTRACT}

Objective: To carry out an epidemiological analysis of suicide, among genders, in the metropolitan region of Maceió, over 20 years (2000 to 2019). Methods: Ecological observational and cross-sectional study, conducted with a sample of 693 suicide victims living in the metropolitan region of Maceió, between both genders. Data from the Ministry of Health's Mortality Information System (SIM), provided by DATASUS platforms, was used as a source of information. Data were analyzed by temporal and spatial descriptive statistics and by Mann-Kendall inferential trend analysis, adopting a significance level of $5 \%$. Results: Of the total number of suicides, $77.5 \%(537)$ were male and $22.5 \%(155)$ were female. The average rate for the metropolitan region was 2.2 per 100,000 population, with 3.3 per 100,000 for men and 1.0 per 100,000 for women. There was a significant increase in rates between 2000 and 2009 for the general population $(p=0.03)$ and for females $(p=0.01)$. Conclusion: Men were the main victims of suicide with higher and more stable rates, for females, while there was a significant increase in rates for females between 2000 and 2009.

Keywords: Time series studies, Suicide, Violence.

\section{RESUMEN}

Objetivo: Realizar un análisis epidemiológico del suicidio, entre géneros, en la región metropolitana de Maceió, durante 20 años (2000 a 2019). Métodos: Se trata de un estudio ecológico observacional y transversal, realizado a partir de 693 víctimas de suicidio residentes en la región metropolitana de Maceió, entre ambos gêneros. Se utilizaron como fuente de información datos del Sistema de Información sobre Mortalidad (SIM) del Ministerio de Salud, proporcionados por las plataformas DATASUS. Los datos fueron analizados por estadística descriptiva temporal y espacial y por análisis de tendencia inferencial de Mann-Kendall, adoptando un nivel de significancia del 5\%. Resultados: Del total de suicidios, $77,5 \%$ (537) fueron hombres y 22,5\% (155) mujeres. La tasa promedio para la región metropolitana fue de 2,2 por 100.000 habitantes, siendo de 3,3 por 100.000 para los hombres y de 1,0 por 100.000 para las mujeres. Hubo un aumento significativo en las tasas entre 2000 y 2009 para la población general $(p=0.03)$ y para las mujeres $(p=0.01)$. Conclusión: Los hombres son las principales víctimas de suicidio y mantienen las tasas más altas y estables, mientras que hubo un aumento significativo en las tasas para las mujeres entre 2000 y 2009.

Palabras clave: Estudios de series studies, Suicide, Violencia.

${ }^{1}$ Centro Universitário Cesmac (CESMAC), Maceió - AL. *E-mail: kevanguilherme@gmail.com

Financiamento da pesquisa: CNPq/MCTIC Edital № 28/2018 (processo no: 408271/2018-3) 


\section{INTRODUÇÃO}

A palavra suicídio passou a ser conhecida no século XVII e seu significado principal é o da morte autoprovocada, sempre associada a inúmeros fatores, desde a vontade própria, honra moral, pecado, crime, até que na segunda metade do século $X X$ passou a ser considerado como doença mental, sendo então possível uma melhor compreensão da problemática (BOTEGA NJ, 2015).

Segunda a Organização Mundial de Saúde (OMS), os principais fatores de risco para o suicídio estão associados aos problemas de saúde mental e abuso de substâncias, à dificuldade de acesso aos serviços de saúde e falta de cuidados necessários, disponibilidade dos meios para cometer o suicídio, reportagem inapropriada de mídia sensacionalista sobre o suicídio, o mito e estigma contra pessoas que procuram ajuda para comportamentos suicidas (WORD HEALTH ORGANIZATION (WHO), 2014).

Dentre os fatores protetores para a tentativa de suicídio destacam-se as relações familiares, as quais possuem um importante papel junto aos pacientes com histórico suicida, uma vez que uma relação saudável entre pais e filhos auxilia na prevenção do suicídio, principalmente quando os pais são responsivos e afetivos (SILVA DA e MARCOLAN JF, 2021; MAGNANI RM e STAUDT AC, 2018).

A taxa mundial de suicídio estimada pela OMS foi de 11,4 óbitos por 100 mil habitantes, sendo 15,0 para homens e 8,0 para mulheres. No Brasil ocorrem cerca de 10 mil mortes por suicídio por ano, com valores estáveis ao longo dos últimos anos (MINISTÉRIO DA SAÚDE, 2017). Na região Nordeste do país, entre os anos 2000 a 2012, houve uma tendência significativa de aumento na taxa de suicídio (72,4\%), apresentando assim o maior crescimento percentual em comparação as demais regiões (MACHADO DB e SANTOS DN, 2015).

No Brasil, o suicídio é registrado pela declaração de óbito emitida por um médico legista de algum instituto médico legal (IML) dos estados. Por se tratar de uma morte violenta, sempre que houver confirmação ou suspeita desse tipo de óbito, faz-se necessário uma investigação da causa do óbito junto a algum IML. Em acordo com o Código de Processo Penal brasileiro é claramente expresso no art. 564, inciso III, alínea b, que haverá nulidade de processos caso não seja feito o exame de corpo de delito, excetuando quando não há possibilidade de realiza-lo por causa justificável (BRASIL, 1941).

Os dados das declarações de óbitos são consolidados no Sistema de Informação de Mortalidade (SIM) do Ministério da Saúde, que retroalimenta as informações recebidas por meio da divulgação de dados agregados por município no site do Departamento de Informática do Sistema Único de Saúde (MINISTÉRIO DA SAÚDE, 2021).

A região de Alagoas situa-se no nordeste brasileiro, sendo historicamente reconhecida como uma região que apresenta crises e problemas estruturais como pobreza, baixa nível educacional e altas taxas de violência urbana, incluindo os homicídios. Apesar disso, ainda há poucos estudos epidemiológicos na literatura explorando o fenômeno do suicídio. Neste contexto, o propósito do presente estudo foi traçar uma análise epidemiológica espaço-temporal acerca do suicídio na região metropolitana de Maceió, ao longo de 20 anos, entre os anos de 2000 até 2019, analisando as diferenças entres os gêneros.

\section{MÉTODOS}

Trata-se de um estudo epidemiológico ecológico observacional retrospectivo, com abordagem de método quantitativo, com investigação do binômio tempo-espaço. De acordo com Almeida-Filho $\mathrm{N}$ e Barreto $\mathrm{ML}$ (2011), os estudos ecológicos são também denominados de agregados e correspondem a um tipo de delineamento de estudo em que a unidade de análise é um conjunto de indivíduos e não indivíduos isolados, daí a denominação estudo "agregado".

Desta maneira, foram revisados 20 anos (2000-2019) de dados agregados relativos ao suicídio para a região metropolitana de Maceió, capital do estado de Alagoas. A região metropolitana de Maceió é composta por outros 12 municípios, além da própria capital: Atalaia, Barra de Santo Antônio, Barra de São Miguel, Coqueiro Seco, Marechal Deodoro, Messias, Murici, Paripueira, Pilar, Rio Largo, Santa Luzia do Norte e Satuba. 
Foram incluídos no estudo apenas vítimas de suicídio na RM com registro no Sistema de Informação de Mortalidade (SIM). O SIM disponibiliza dados agregados, a partir do qual foi construída uma série temporal e uma distribuição espacial dos casos de suicídio. Para acessar os dados oriundos do SIM, disponibilizados pelo Departamento de Informática do Sistema Único de Saúde (DATASUS), utilizou-se alguns filtros de busca para inclusão no estudo, estes filtros foram delimitados para acesso às informações de suicídio na plataforma TabWin do DATASUS (MINISTÉRIO DA SAÚDE, 2021).

A sequência de acesso na base do SIM foi a seguinte: Estatísticas Vitais > Mortalidade pela CID > Óbitos por Causas Externas > Alagoas. Na linha foram marcados os municípios de Alagoas, sendo posteriormente separados aqueles da região metropolitana dos demais. Em grande grupo CID foram consultados os dados de mortalidade pela causa CID "Lesões Autoprovocadas Intencionalmente", que envolve os códigos X60 até X84, em conformidade com a Classificação Internacional de Doenças-Versão 10, no seu Capítulo XX (INTERNATIONAL CLASSIFICATION OF DISEASE AND RELATED HEALTH PROBLEMS, 2016). Utilizamos os óbitos por residência dentre o período de 20 anos, compreendidos entre os anos 2000 a 2019, estratificados segundo os gêneros. Para este estudo, a variável desfecho foi o óbito por suicídio entre os gêneros.

Para a coleta realizada via SIM foram utilizados como critérios de inclusão para este estudo: óbitos registrados como suicídio, notificados no município de Maceió, disponíveis no SIM/DATASUS entre os anos 2000 a 2019. A escolha destes anos se deve ao fato de serem dados representativos de vinte anos, um tempo suficiente para observar padrões de variação ao longo do tempo. Além disso, estes são os dados oficiais nacionais reportados ao Ministério da Saúde. Durante a fase de coleta de dados o ano de 2020 não se apresentava consolidado no SIM, motivo pelo qual não foi incluído na presente análise.

A análise de série temporal inicialmente foi feita a partir da descrição do número absoluto dos casos de suicídio por município da região metropolitana de Maceió, estratificados pelo gênero da vítima. Em seguida foram computadas as taxas de suicídio geral por 100.000 habitantes e as taxas médias de todos os municípios da região metropolitana. Foi utilizado a estimativa populacional anual do Ministério da Saúde (2020).

As séries temporais de suicídio geral, suicídio entre homens e suicídio entre mulheres foram suavizadas pelo método de suavização exponencial. A análise de tendência de série foi feita através do teste de MannKendall, que testou as hipóteses alternativas e nulas para tendência crescente e decrescente. Em todas as análises foi adotado um nível de significância de $5 \%$. Foram utilizados os programas IBM SPSS ${ }^{\circledR}$ Statistics (versão 20.0), Microsoft Excel ${ }^{\circledR} 2016$ e Minitab $^{\circledR}$ (versão 19.0) para realização das análise e confecções dos gráficos.

A análise da série espacial foi realizada a partir da distribuição dos casos por município de residência das vítimas de suicídio, seguido pela distribuição espacial das taxas. Foi utilizado o programa ArcGis (versão 10.4) e ArcMap (versão 10.4) para confecção dos mapas.

\section{RESULTADOS}

Dentre os 693 casos de suicídio registrados ao longo de 20 anos na RM de Maceió, 537 (77,5\%) acometeram os homens, e $155(22,5 \%)$ as mulheres. Houve ainda um dado sem registro para o gênero. A Tabela 1 abaixo exibe o número absoluto dos casos destacando a capital do estado que concentrou um maior quantitativo.

$\mathrm{Na}$ Tabela 2 temos o quantitativo das taxas com ajuste para o tamanho populacional de cada município da RM, expressos em função da base para 100.000 habitantes. A taxa média de suicídio para pessoas residentes da região metropolitana foi de 2,2 por 100.000 hab., sendo 3,3 por 100.000 hab. entre homens e 1,0 por 100.000 hab. em mulheres. As taxas de suicídio na população de homens foram superiores às das mulheres em todos os anos da série. Os municípios com maiores taxas médias foram Barra de São Miguel e Maceió, 5,1 por 100.000 hab. e 3,0 por 100.000 hab. respectivamente. O município de Barra de São Miguel apresentou picos de incidência entre os anos de 2014 e 2017, e muitos municípios apresentaram sucessivos anos com taxas nulas. 
Tabela 1 - Evolução total do número absoluto de suicídios, $n=693$. Região metropolitana de Maceió - AL, 2000-2019.

\begin{tabular}{|c|c|c|c|c|c|c|c|c|c|c|c|c|c|c|c|c|c|c|c|c|}
\hline ஓे & ฉి & ฐิి & ฉิ & ธి & ఫ్ & ฉి & ๕ั้ & ธิธิ & 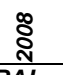 & ฉి & กิ & กิ & ลิ & $\stackrel{m}{\grave{n}}$ & $\frac{\pi}{2}$ & $\stackrel{\text { ñ }}{\text { ลे }}$ & $\stackrel{0}{\grave{2}}$ & $\hat{\text { ลे }}$ & $\stackrel{\infty}{\grave{2}}$ & ลे \\
\hline \multirow{2}{*}{\multicolumn{21}{|c|}{ POPULAÇÃO GERAL }} \\
\hline \multicolumn{4}{|l|}{ MUNICIPIO } & & & & & & & & & & & & & & & & & \\
\hline Atalaia & 0 & 1 & 1 & 1 & 2 & 0 & 0 & 0 & 0 & 1 & 1 & 1 & 0 & 1 & 0 & 1 & 0 & 0 & 0 & 0 \\
\hline B. de Sto. Antônio & 1 & 0 & 0 & 0 & 0 & 1 & 1 & 0 & 0 & 0 & 0 & 0 & 1 & 0 & 0 & 0 & 0 & 0 & 0 & 0 \\
\hline B. de São Miguel & 0 & 0 & 0 & 1 & 0 & 0 & 0 & 1 & 0 & 0 & 0 & 0 & 0 & 0 & 2 & 1 & 2 & 1 & 0 & 0 \\
\hline Coqueiro Seco & 0 & 0 & 0 & 0 & 1 & 0 & 0 & 0 & 1 & 0 & 0 & 0 & 0 & 0 & 0 & 0 & 0 & 0 & 0 & 0 \\
\hline Maceió & 12 & 34 & 14 & 18 & 24 & 16 & 31 & 29 & 39 & 35 & 29 & 31 & 29 & 37 & 34 & 32 & 30 & 23 & 36 & 36 \\
\hline Marechal Deodoro & 0 & 1 & 0 & 0 & 2 & 0 & 0 & 0 & 3 & 2 & 3 & 1 & 3 & 1 & 1 & 0 & 1 & 2 & 1 & 0 \\
\hline Messias & 0 & 1 & 0 & 0 & $\overline{0}$ & 0 & 0 & 0 & 0 & 1 & 0 & 0 & 0 & 0 & 1 & 0 & 0 & 0 & 1 & 0 \\
\hline Murici & 0 & 0 & 0 & 0 & 0 & 0 & 1 & 0 & 3 & 0 & 0 & 0 & 0 & 0 & 0 & 1 & 2 & 0 & 1 & 1 \\
\hline Paripueira & 0 & 0 & 0 & 0 & 0 & 1 & 1 & 0 & 0 & 0 & 0 & 1 & 0 & 0 & 0 & 0 & 0 & 2 & 0 & 0 \\
\hline Pilar & 0 & 0 & 0 & 0 & 1 & 0 & 1 & 2 & 0 & 4 & 2 & 3 & 0 & 4 & 0 & 0 & 1 & 0 & 1 & 1 \\
\hline Rio Largo & 0 & 2 & 2 & 0 & 3 & 1 & 1 & 3 & 1 & 3 & 0 & 3 & 2 & 3 & 1 & 1 & 2 & 3 & 1 & 2 \\
\hline Sta. Luzia do Norte & 0 & 0 & 0 & 0 & 0 & 0 & 1 & 0 & 0 & 0 & 0 & 0 & 0 & 0 & 0 & 0 & 0 & 0 & 0 & 0 \\
\hline Satuba & 0 & 1 & 0 & 0 & 1 & 0 & 0 & 0 & 1 & 0 & 1 & 0 & 0 & 0 & 0 & 1 & 0 & 1 & 0 & 0 \\
\hline TOTAL & 13 & 40 & 17 & 20 & 34 & 19 & 37 & 35 & 48 & 46 & 36 & 40 & 35 & 46 & 39 & 37 & 38 & 32 & 41 & 40 \\
\hline \multirow{2}{*}{\multicolumn{21}{|c|}{ GÊNERO MASCULINO }} \\
\hline MUNICIPIO & & & & & & & & & & & & & & & & & & & & \\
\hline Atalaia & 0 & 1 & 1 & 1 & 1 & 0 & 0 & 0 & 0 & 1 & 0 & 1 & 0 & 1 & 0 & 0 & 0 & 0 & 0 & 0 \\
\hline B. de Sto. Antônio & 1 & 0 & 0 & 0 & 0 & 1 & 1 & 0 & 0 & 0 & 0 & 0 & 1 & 0 & 0 & 0 & 0 & 0 & 0 & 0 \\
\hline B. de São Miguel & 0 & 0 & 0 & 1 & 0 & 0 & 0 & 1 & 0 & 0 & 0 & 0 & 0 & 0 & 0 & 1 & 1 & 1 & 0 & 0 \\
\hline Coqueiro Seco & 0 & 0 & 0 & 0 & 1 & 0 & 0 & 0 & 1 & 0 & 0 & 0 & 0 & 0 & 0 & 0 & 0 & 0 & 0 & 0 \\
\hline Maceió & 12 & 31 & 11 & 15 & 24 & 11 & 23 & 25 & 34 & 29 & 22 & 20 & 19 & 29 & 27 & 27 & 17 & 17 & 26 & 23 \\
\hline Marechal Deodoro & 0 & 1 & 0 & 0 & 2 & 0 & 0 & 0 & 3 & 1 & 3 & 1 & 2 & 0 & 1 & 0 & 1 & 2 & 1 & 0 \\
\hline Messias & 0 & 1 & 0 & 0 & 0 & 0 & 0 & 0 & 0 & 1 & 0 & 0 & 0 & 0 & 1 & 0 & 0 & 0 & 1 & 0 \\
\hline Murici & 0 & 0 & 0 & 0 & 0 & 0 & 1 & 0 & 2 & 0 & 0 & 0 & 0 & 0 & 0 & 1 & 1 & 0 & 1 & 1 \\
\hline Paripueira & 0 & 0 & 0 & 0 & 0 & 0 & 0 & 0 & 0 & 0 & 0 & 1 & 0 & 0 & 0 & 0 & 0 & 1 & 0 & 0 \\
\hline Pilar & 0 & 0 & 0 & 0 & 1 & 0 & 1 & 2 & 0 & 3 & 2 & 3 & 0 & 2 & 0 & 0 & 1 & 0 & 1 & 1 \\
\hline Rio Largo & 0 & 2 & 1 & 0 & 2 & 1 & 1 & 3 & 1 & 1 & 0 & 2 & 1 & 2 & 1 & 0 & 2 & 3 & 0 & 1 \\
\hline Sta. Luzia do Norte & 0 & 0 & 0 & 0 & 0 & 0 & 0 & 0 & 0 & 0 & 0 & 0 & 0 & 0 & 0 & 0 & 0 & 0 & 0 & 0 \\
\hline Satuba & 0 & 0 & 0 & 0 & 1 & 0 & 0 & 0 & 1 & 0 & 1 & 0 & 0 & 0 & 0 & 1 & 0 & 1 & 0 & 0 \\
\hline TOTAL & 13 & 36 & 13 & 17 & 32 & 13 & 27 & 31 & 42 & 36 & 28 & 28 & 23 & 34 & 30 & 30 & 23 & 25 & 30 & 26 \\
\hline \multirow{2}{*}{\multicolumn{21}{|c|}{ GÊNNERO FEMININO }} \\
\hline MUNICÍPIO & & & & & & & & & & & & & & & & & & & & \\
\hline Atalaia & 0 & 0 & 0 & 0 & 1 & 0 & 0 & 0 & 0 & 0 & 1 & 0 & 0 & 0 & 0 & 1 & 0 & 0 & 0 & 0 \\
\hline B. de Sto. Antônio & 0 & 0 & 0 & 0 & 0 & 0 & 0 & 0 & 0 & 0 & 0 & 0 & 0 & 0 & 0 & 0 & 0 & 0 & 0 & 0 \\
\hline B. de São Miguel & 0 & 0 & 0 & 0 & 0 & 0 & 0 & 0 & 0 & 0 & 0 & 0 & 0 & 0 & 2 & 0 & 1 & 0 & 0 & 0 \\
\hline Coqueiro Seco & 0 & 0 & 0 & 0 & 0 & 0 & 0 & 0 & 0 & 0 & 0 & 0 & 0 & 0 & 0 & 0 & 0 & 0 & 0 & 0 \\
\hline Maceió & 0 & 3 & 3 & 3 & 0 & 5 & 8 & 4 & 5 & 6 & 7 & 11 & 10 & 8 & 7 & 5 & 13 & 6 & 10 & 13 \\
\hline Marechal Deodoro & 0 & 0 & 0 & 0 & 0 & 0 & 0 & 0 & 0 & 1 & 0 & 0 & 1 & 1 & 0 & 0 & 0 & 0 & 0 & 0 \\
\hline Messias & 0 & 0 & 0 & 0 & 0 & 0 & 0 & 0 & 0 & 0 & 0 & 0 & 0 & 0 & 0 & 0 & 0 & 0 & 0 & 0 \\
\hline Murici & 0 & 0 & 0 & 0 & 0 & 0 & 0 & 0 & 1 & 0 & 0 & 0 & 0 & 0 & 0 & 0 & 1 & 0 & 0 & 0 \\
\hline Paripueira & 0 & 0 & 0 & 0 & 0 & 1 & 1 & 0 & 0 & 0 & 0 & 0 & 0 & 0 & 0 & 0 & 0 & 1 & 0 & 0 \\
\hline Pilar & 0 & 0 & 0 & 0 & 0 & 0 & 0 & 0 & 0 & 1 & 0 & 0 & 0 & 2 & 0 & 0 & 0 & 0 & 0 & 0 \\
\hline Rio Largo & 0 & 0 & 1 & 0 & 1 & 0 & 0 & 0 & 0 & 2 & 0 & 1 & 1 & 1 & 0 & 1 & 0 & 0 & 1 & 1 \\
\hline Sta. Luzia do Norte & 0 & 0 & 0 & 0 & 0 & 0 & 1 & 0 & 0 & 0 & 0 & 0 & 0 & 0 & 0 & 0 & 0 & 0 & 0 & 0 \\
\hline Satuba & 0 & 0 & 0 & 0 & 0 & 0 & 0 & 0 & 0 & 0 & 0 & 0 & 0 & 0 & 0 & 0 & 0 & 0 & 0 & 0 \\
\hline TOTAL & 0 & 3 & 4 & 3 & 2 & 6 & 10 & 4 & 6 & 10 & 8 & 12 & 12 & 12 & 9 & 7 & 15 & 7 & 11 & 14 \\
\hline
\end{tabular}

Fonte: Silva AV, et al., 2021; dados extraídos do DATASUS. 
Tabela 2 - Evolução total das taxas de suicídio por 100.000 habitantes, $n=693$. Região metropolitana de Maceió - AL, 2000-2019.

\begin{tabular}{|c|c|c|c|c|c|c|c|c|c|c|c|c|c|c|c|c|c|c|c|c|c|}
\hline$\stackrel{\circ}{\frac{\pi}{4}}$ & ષ્సి & $\overline{\text { ర్ }}$ & ণิ & ర్సి & ષ্ণ & ֻั & ષัర & ర్సి & ֻ & ఫ్ & 울 & $\overline{\text { స }}$ & $\stackrel{N}{\text { N }}$ & $\sum_{\text {N }}^{\infty}$ & $\stackrel{+}{\text { N }}$ & $\sum_{\text {N }}^{10}$ & $\stackrel{0}{\grave{N}}$ & $\hat{\mathrm{N}}$ & $\stackrel{\infty}{\stackrel{\infty}{N}}$ & 올 & 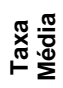 \\
\hline \multicolumn{22}{|c|}{ POPULAÇÃO GERAL } \\
\hline Atalaia & 0,0 & 2,4 & 2.4 & 2,3 & 4.6 & 0,0 & 0,0 & 0,0 & 0,0 & 2,2 & 2,2 & 2.2 & 0,0 & 2.2 & 0,0 & 22 & 00 & 00 & 00 & 00 & 11 \\
\hline B. de Sto. Antônio & $\begin{array}{l}8,0 \\
8,6\end{array}$ & $\begin{array}{l},, 4 \\
0,0\end{array}$ & 0,0 & 0,0 & 0,0 & 7,5 & 7,4 & 0,0 & 0,0 & 0,0 & 0,0 & 0,0 & 6,7 & 0,0 & 0,0 & $\begin{array}{l}2,2 \\
0,0\end{array}$ & 0,0 & 0,0 & 0,0 & 0,0 & 1,5 \\
\hline B. de São Miguel & 0,0 & 0,0 & 0,0 & 14,3 & 0,0 & 0,0 & 0,0 & 13,4 & 0,0 & 0,0 & 0,0 & 0,0 & 0,0 & 0,0 & 24,9 & 12,4 & 24,5 & 12,2 & 0,0 & 0,0 & 5,1 \\
\hline Coqueiro Seco & 0,0 & 0,0 & 0,0 & 0,0 & 18,4 & 0,0 & 0,0 & 0,0 & 17,9 & 0,0 & 0,0 & 0,0 & 0,0 & 0,0 & 0,0 & 0,0 & 0,0 & 0,0 & 0,0 & 0,0 & 1,8 \\
\hline Maceió & 1,5 & 4,1 & 1,6 & 2,1 & 2,7 & 1,8 & 3,4 & 3,1 & 4,2 & 3,7 & 3,0 & 3,2 & 3,0 & 3,8 & 3,4 & 3,2 & 3,0 & 2,3 & 3,6 & 3,5 & 3,0 \\
\hline Marechal Deodoro & 0,0 & 2,6 & 0,0 & 0,0 & 4,8 & 0,0 & 0,0 & 0,0 & 6,6 & 4,3 & 6,4 & 2,1 & 6,2 & 2,0 & $\begin{array}{l}2,4 \\
2,0\end{array}$ & 0,0 & 2,0 & 3,9 & 1,9 & 0,0 & 2,2 \\
\hline Messias & 0,0 & 7,8 & 0,0 & 0,0 & 0,0 & 0,0 & 0,0 & 0,0 & 0,0 & $\begin{array}{l}4,0 \\
6,3\end{array}$ & $\begin{array}{l}0,4 \\
0,0\end{array}$ & 0,0 & $\begin{array}{l}0,0 \\
0,0\end{array}$ & 0,0 & $\begin{array}{l}5,0 \\
5,9\end{array}$ & 0,0 & 0,0 & 0,0 & 5,7 & 0,0 & 1,3 \\
\hline Murici & $\begin{array}{l}0,0 \\
0,0\end{array}$ & $\begin{array}{l}1,0 \\
0,0\end{array}$ & 0,0 & 0,0 & $\begin{array}{l}0,0 \\
0,0\end{array}$ & $\begin{array}{l}0,0 \\
0,0\end{array}$ & 3,8 & 0,0 & 11,1 & $\begin{array}{l}0,0 \\
0,0\end{array}$ & $\begin{array}{l}0,0 \\
0,0\end{array}$ & 0,0 & $\begin{array}{l}0,0 \\
0,0\end{array}$ & $\begin{array}{l}0,0 \\
0,0\end{array}$ & $\begin{array}{l}0,0 \\
0,0\end{array}$ & $\begin{array}{l}0,0 \\
3,6\end{array}$ & $\begin{array}{l}7,0 \\
7,2\end{array}$ & 0,0 & $\begin{array}{l}3,6 \\
3,6\end{array}$ & $\begin{array}{l}3,0 \\
3,5\end{array}$ & $\begin{array}{l}1,0 \\
1,6\end{array}$ \\
\hline Paripueira & 0,0 & 0,0 & 0,0 & 0,0 & 0,0 & $\begin{array}{l}9,9 \\
9,9\end{array}$ & $\begin{array}{l}9,0 \\
9,5\end{array}$ & 0,0 & 0,0 & 0,0 & $\begin{array}{l}0,0 \\
0,0\end{array}$ & 8,5 & $\begin{array}{l}0,0 \\
0,0\end{array}$ & $\begin{array}{l}0,0 \\
0,0\end{array}$ & $\begin{array}{l}0,0 \\
0,0\end{array}$ & $\begin{array}{l}3,0 \\
0,0\end{array}$ & $\begin{array}{l}, 2 \\
0,0\end{array}$ & $\begin{array}{l}0,0 \\
15,6\end{array}$ & $\begin{array}{l}3,6 \\
0,0\end{array}$ & $\begin{array}{l}3,5 \\
0,0\end{array}$ & $\begin{array}{l}1,6 \\
2,2\end{array}$ \\
\hline & $\begin{array}{l}0,0 \\
0,0\end{array}$ & 0,0 & $\begin{array}{l}0,0 \\
0,0\end{array}$ & 0,0 & $\begin{array}{l}3,0 \\
3,0\end{array}$ & $\begin{array}{l}, 9 \\
0,0\end{array}$ & $\begin{array}{l}3,0 \\
3,0\end{array}$ & $\begin{array}{l}6,0 \\
6,0\end{array}$ & 0,0 & 11,8 & $\begin{array}{l}5,9 \\
5\end{array}$ & $\begin{array}{l}8,5 \\
8,8\end{array}$ & 0,0 & 11,6 & $\begin{array}{l}0,0 \\
0,0\end{array}$ & 0,0 & $\begin{array}{l}2,0 \\
2,9\end{array}$ & $\begin{array}{l}0,0 \\
0,0\end{array}$ & $\begin{array}{l}2,9 \\
2,9\end{array}$ & $\begin{array}{l}2,8 \\
2,8\end{array}$ & $\begin{array}{l}2,2 \\
2,9\end{array}$ \\
\hline Rio Largo & 0,0 & 3,1 & 3,0 & 0,0 & 4,5 & 1,5 & 1,5 & $\begin{array}{l}4,0 \\
4,4\end{array}$ & 1,4 & 4,3 & 0,0 & $\begin{array}{l}\quad, 0 \\
4,3\end{array}$ & $\begin{array}{l}2,7 \\
2,7\end{array}$ & 4,1 & 1,4 & 1,3 & 2,7 & 4,0 & 1,3 & $\begin{array}{l}2,0 \\
2,7\end{array}$ & 2,4 \\
\hline Sta. Luzia do Norte & 0,0 & 0,0 & 0,0 & 0,0 & 0,0 & 0,0 & 14,5 & 0,0 & 0,0 & 0,0 & 0,0 & 0,0 & 0,0 & 0,0 & 0,0 & 0,0 & 0,0 & 0,0 & 0,0 & 0,0 & 0,7 \\
\hline $\begin{array}{l}\text { Satuba } \\
\end{array}$ & 0,0 & 8,0 & 0,0 & 0,0 & 7,4 & 0,0 & 0,0 & 0,0 & 6,9 & 0,0 & 6,7 & 0,0 & 0,0 & 0,0 & 0,0 & 7,6 & 0,0 & 7,4 & 0,0 & 0,0 & 2,2 \\
\hline TOTAL & 0,8 & 2,2 & 0,5 & 1,4 & 3,5 & 1,6 & 3,3 & 2,1 & 3,7 & 2,5 & 1,9 & 2,2 & 1,4 & 1,8 & 2,9 & 2,3 & 3,3 & 3,5 & 1,5 & 1,0 & 2,2 \\
\hline \multirow{2}{*}{\multicolumn{22}{|c|}{ GENNERO MASCULINO }} \\
\hline \multicolumn{3}{|c|}{ GEIVERU IMIASCULIVU } & & & & & & & & & & & & & & & & & & & \\
\hline Atalaia & 0,0 & 4,7 & 4,6 & 4,6 & 4,6 & 0,0 & 0,0 & 0,0 & 0,0 & 4,4 & 0,0 & 4,4 & 0,0 & 4,4 & 0,0 & 0,0 & 0,0 & 0,0 & 0,0 & 0,0 & 1,6 \\
\hline B. de Sto. Antônio & 17,0 & 0,0 & 0,0 & 0,0 & 0,0 & 15,0 & 14,7 & 0,0 & 0,0 & 0,0 & 0,0 & 0,0 & 13,5 & 0,0 & 0,0 & 0,0 & 0,0 & 0,0 & 0,0 & 0,0 & 3,0 \\
\hline B. de São Miguel & 0,0 & 0,0 & 0,0 & 28,8 & 0,0 & 0,0 & 0,0 & 27,0 & 0,0 & 0,0 & 0,0 & 0,0 & 0,0 & 0,0 & 0,0 & 24,9 & 24.8 & 24.6 & 0,0 & 0,0 & 6,5 \\
\hline Coqueiro Seco & 0,0 & 0,0 & 0,0 & 0,0 & 36,5 & 0,0 & 0,0 & 0,0 & 35,8 & 0,0 & 0,0 & 0,0 & 0,0 & 0,0 & 0,0 & 0,0 & $\begin{array}{l}2,0 \\
0,0\end{array}$ & 0,0 & 0,0 & 0,0 & 3,6 \\
\hline Maceió & 3,1 & 7,9 & 2,7 & 3,7 & 5,8 & 2,6 & 5,4 & 5,8 & 7,8 & 6,5 & 4,9 & 4,4 & 4,2 & 6,4 & 5,9 & 5,8 & 3,7 & 3,6 & 5,5 & 4,9 & $\begin{array}{l}5,0 \\
5,0\end{array}$ \\
\hline Marechal Deodoro & 0,0 & 5,3 & 0,0 & 0,0 & 9,7 & 0,0 & 0,0 & 0,0 & 13,4 & 4,4 & 12,9 & 4,2 & 8,4 & 0,0 & 4,1 & 0,0 & $\begin{array}{l}4,0 \\
4,0\end{array}$ & 8,0 & 4,0 & 0,0 & 3,9 \\
\hline Messias & 0,0 & $\begin{array}{l}5,0 \\
15,7\end{array}$ & $\begin{array}{l}0,0 \\
0,0\end{array}$ & $\begin{array}{l}0,0 \\
0,0\end{array}$ & $\begin{array}{l}, l \\
0,0\end{array}$ & $\begin{array}{l}0,0 \\
0,0\end{array}$ & $\begin{array}{l}0,0 \\
0,0\end{array}$ & 0,0 & $\begin{array}{l}1,4 \\
0,0\end{array}$ & $\begin{array}{l}4,4 \\
12,9\end{array}$ & 0,0 & $\begin{array}{l}4,2 \\
0,0\end{array}$ & $\begin{array}{l}0,4 \\
0,0\end{array}$ & $\begin{array}{l}0,0 \\
0,0\end{array}$ & $\begin{array}{l}4,1 \\
12,1\end{array}$ & 0,0 & $\begin{array}{l}\begin{array}{l}4,0 \\
0,0\end{array} \\
-\end{array}$ & $\begin{array}{l}0,0 \\
0,0\end{array}$ & $\begin{array}{l}4,0 \\
11,7\end{array}$ & $\begin{array}{l}0,0 \\
0,0\end{array}$ & $\begin{array}{l}2,9 \\
2,6\end{array}$ \\
\hline Murici & 0,0 & 0,0 & 0,0 & 0,0 & 0,0 & 0,0 & 7,5 & 0,0 & 14,9 & 0,0 & 0,0 & 0,0 & 0,0 & 0,0 & 0,0 & 7,3 & 7,2 & 0,0 & 7,2 & 7,2 & 2,0 \\
\hline Paripueira & 0,0 & 0,0 & 0,0 & 0,0 & 0,0 & 0,0 & 0,0 & 0,0 & 0,0 & 0,0 & 0,0 & 17,2 & 0,0 & 0,0 & 0,0 & 0,0 & 0,0 & 15,8 & 0,0 & 0,0 & 1,6 \\
\hline Pilar & 0,0 & 0,0 & 0,0 & 0,0 & 6,2 & 0,0 & 6,1 & 12,2 & 0,0 & 18,2 & 12,1 & 18,1 & 0,0 & 12,0 & 0,0 & 0,0 & 6,0 & 0,0 & 6,0 & 6,0 & 5,1 \\
\hline Rio Largo & 0,0 & 6,2 & 3,1 & 0,0 & 6,1 & 3,0 & 3,0 & 8,9 & 3,0 & 2,9 & 0,0 & 5,8 & 2,8 & 5,6 & 2,8 & 0,0 & 5,6 & 8,3 & 0,0 & 2,8 & 3,5 \\
\hline Sta. Luzia do Norte & 0,0 & 0,0 & 0,0 & 0,0 & 0,0 & 0,0 & 0,0 & 0,0 & 0,0 & 0,0 & 0,0 & 0,0 & 0,0 & 0,0 & 0,0 & 0,0 & 0,0 & 0,0 & 0,0 & 0,0 & 0,0 \\
\hline Satuba & 0,0 & 0,0 & 0,0 & 0,0 & 15,2 & 0,0 & 0,0 & 0,0 & 14,1 & 0,0 & 13,7 & 0,0 & 0,0 & 0,0 & 0,0 & 15,8 & 0,0 & 15,2 & 0,0 & 0,0 & $\begin{array}{l}3,7 \\
3,7\end{array}$ \\
\hline TOTAL & 1,5 & 3,1 & 0,8 & 2,9 & 6,5 & 1,6 & 2,8 & 4,1 & 6,8 & 3,8 & 3,4 & 4,2 & 2,2 & $\begin{array}{l}2,2 \\
\end{array}$ & 1,9 & 4,1 & 3,9 & 5,8 & 2,6 & 1,6 & $\begin{array}{l}3,1 \\
3,3\end{array}$ \\
\hline \multicolumn{22}{|c|}{ GENERO FEMININO } \\
\hline MUNICIPIO & & & & & & & & & & & & & & & & & & & & & \\
\hline Atalaia & 0,0 & 0,0 & 0,0 & 0,0 & 4,7 & 0,0 & 0,0 & 0,0 & 0,0 & 0,0 & 4,4 & 0,0 & 0,0 & 0,0 & 0,0 & 4,3 & 0,0 & 0,0 & 0,0 & 0,0 & 0,7 \\
\hline B. de Sto. Antônio & 0,0 & 0,0 & 0,0 & 0,0 & 0,0 & 0,0 & 0,0 & 0,0 & 0,0 & 0,0 & 0,0 & 0,0 & 0,0 & 0,0 & 0,0 & 0,0 & 0,0 & 0,0 & 0,0 & 0,0 & 0,0 \\
\hline B. de São Miguel & 0,0 & 0,0 & 0,0 & 0,0 & 0 & $\begin{array}{l}0,0 \\
0,0\end{array}$ & 0,0 & $\begin{array}{l}0,0 \\
0,0\end{array}$ & 0,0 & 0,0 & 0,0 & 0,0 & 0,0 & 0,0 & 49,4 & 0,0 & 24,3 & $\begin{array}{l}0,0 \\
0,0\end{array}$ & 0,0 & 0,0 & $\begin{array}{l}3,0 \\
3,7\end{array}$ \\
\hline Coqueiro Seco & 0,0 & 0,0 & 0,0 & 0,0 & 0,0 & 0,0 & 0,0 & 0,0 & 0,0 & 0,0 & 0,0 & 0,0 & 0,0 & 0,0 & $\begin{array}{l}3,4 \\
0,0\end{array}$ & 0,0 & 0,0 & 0,0 & 0,0 & 0,0 & 0,0 \\
\hline Maceió & 0,0 & 0,7 & 0,7 & 0,7 & 0,0 & 1,1 & 1,7 & 0,8 & 1,0 & 1,2 & 1,4 & 2,1 & 1,9 & 1,5 & 1,3 & 0,9 & 2,4 & 1,1 & 1,8 & 2,4 & 1,2 \\
\hline Marechal Deodoro & 0,0 & 0,0 & 0,0 & 0,0 & 0,0 & 0,0 & 0,0 & 0,0 & 0,0 & 4,3 & 0,0 & 0,0 & 4,1 & 4,0 & 0,0 & 0,0 & 0,0 & 0,0 & 0,0 & $\begin{array}{l}2,4 \\
0,0\end{array}$ & 0,6 \\
\hline Messias & 0,0 & 0,0 & 0,0 & 0,0 & 0,0 & 0,0 & 0,0 & 0,0 & 0,0 & 0,0 & 0,0 & 0,0 & 0,0 & 0,0 & 0,0 & 0,0 & 0,0 & 0,0 & 0,0 & 0,0 & 0,0 \\
\hline Murici & 0,0 & 0,0 & 0,0 & 0,0 & 0,0 & 0,0 & 0,0 & 0,0 & 7,4 & 0,0 & 0,0 & 0,0 & 0,0 & 0,0 & 0,0 & 0,0 & 7,1 & 0,0 & 0,0 & 0,0 & 0,7 \\
\hline Paripueira & 0,0 & 0,0 & 0,0 & 0,0 & 0,0 & 19,4 & 18,8 & 0,0 & 0,0 & 0,0 & 0,0 & 0,0 & 0,0 & 0,0 & 0,0 & 0,0 & 0.0 & 15,4 & 0,0 & 0,0 & 2,7 \\
\hline Pilar & 0,0 & 0,0 & 0,0 & 0,0 & 0,0 & 0,0 & 0,0 & 0,0 & 0,0 & 5,7 & 0,0 & 0,0 & 0,0 & 11,2 & 0,0 & 0,0 & 0,0 & $\begin{array}{l}0,0 \\
0,0\end{array}$ & 0,0 & 0,0 & 0,8 \\
\hline Rio Largo & 0,0 & 0,0 & $\begin{array}{l}3,0 \\
3,0\end{array}$ & 0,0 & $\begin{array}{l}2,0 \\
2,9\end{array}$ & $\begin{array}{l}0,0 \\
0,0\end{array}$ & 0,0 & $\begin{array}{l}0,0 \\
0,0\end{array}$ & 0,0 & 5,6 & 0,0 & 2,8 & $\begin{array}{l}2,7 \\
2,7\end{array}$ & 2,6 & 0,0 & 2,6 & $\begin{array}{l}0,0 \\
0,0\end{array}$ & $\begin{array}{l}0,0 \\
0,0\end{array}$ & 2,6 & $\begin{array}{l}2,0 \\
2,6\end{array}$ & $\begin{array}{l}, 0 \\
1,4\end{array}$ \\
\hline Sta. Luzia do Norte & 0,0 & 0,0 & 0,0 & 0,0 & 0,0 & 0,0 & 28,7 & 0,0 & 0,0 & 0,0 & 0,0 & 0 & 0,0 & 0,0 & 0,0 & 0,0 & 0,0 & 0,0 & 0,0 & 0,0 & $\begin{array}{l}1,4 \\
1,4\end{array}$ \\
\hline $\begin{array}{l}\text { Satuba } \\
\end{array}$ & 0,0 & 0,0 & 0,0 & 0,0 & 0,0 & 0,0 & 0,0 & 0,0 & 0,0 & 0,0 & 0,0 & 0,0 & 0,0 & 0,0 & 0,0 & 0,0 & 0,0 & 0,0 & 0,0 & 0,0 & 0,0 \\
\hline TOTAL & 0,0 & 0,1 & 0,3 & 0,1 & 0,6 & 1,6 & 3,8 & 0,1 & 0,6 & 1,3 & 0,4 & 0,4 & 0,7 & 1,5 & 3,9 & 0,6 & 2,6 & 1,3 & 0,3 & $\begin{array}{l}0,4 \\
0\end{array}$ & $\begin{array}{l}1,0 \\
1,0\end{array}$ \\
\hline
\end{tabular}

Fonte: Silva AV, et al., 2021; dados extraídos do DATASUS. 
Observando a curva das taxas de suicídio identificadas na Figura 1 abaixo é possível perceber que visualmente há uma tendência crescente de suicídio para a população geral. O gráfico específico por gênero da vítima demonstra um comportamento de maior inclinação da curva para o gênero feminino, notadamente nos primeiros 10 anos da série.

Figura 1 - Tendência temporal do suicídio geral e dentre os gêneros para a região metropolitana de Maceió. Suicidio geral
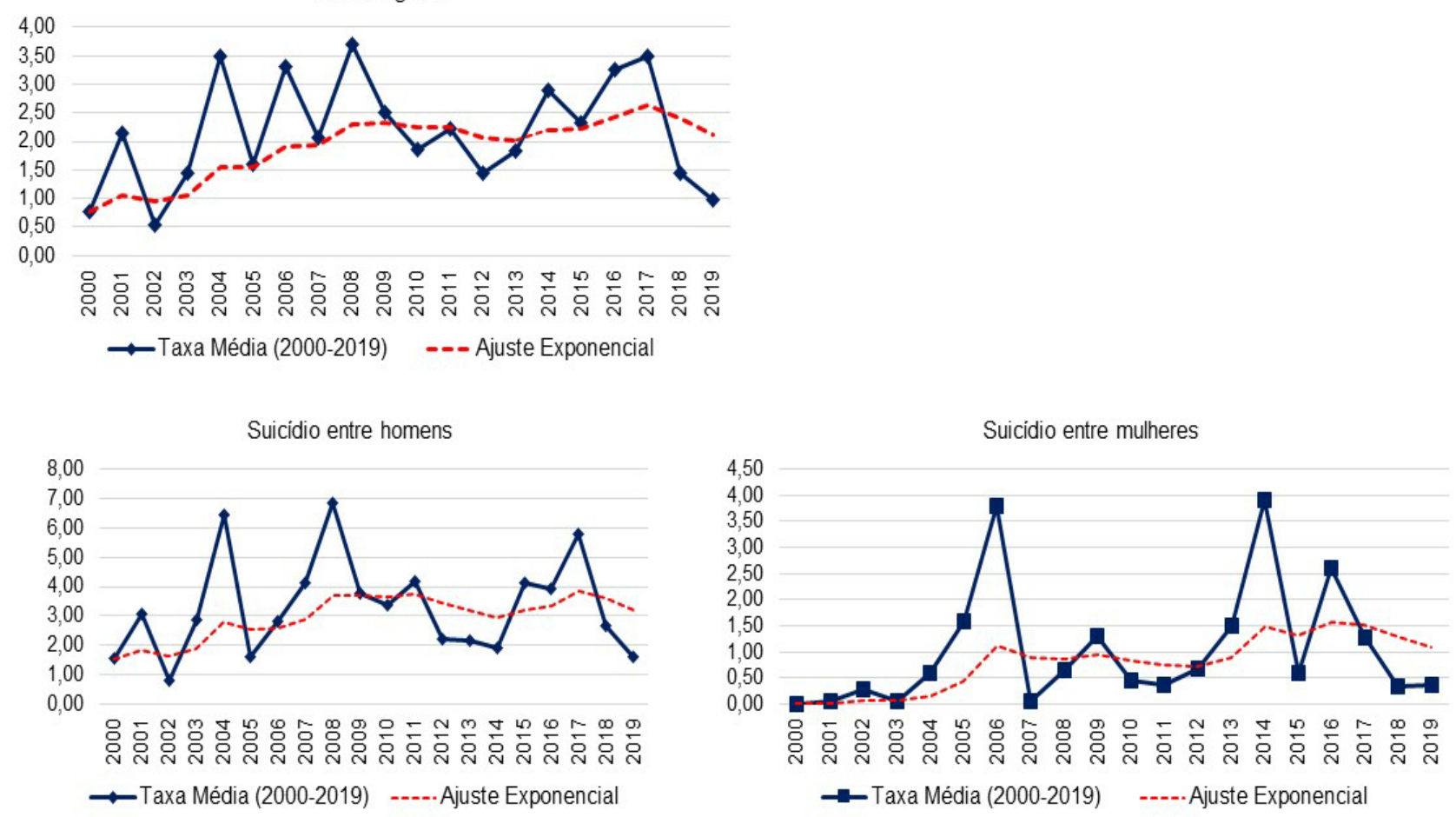

Fonte: Silva AV, et al., 2021; dados extraídos do DATASUS.

A análise de tendência realizada pelo teste de Mann-Kendall confirmou que houve tendência crescente de suicídio entre os anos de 2000 a 2009 para a população geral (homens e mulheres agrupados). A decomposição da série pelos gêneros mostrou que a tendência foi observada apenas para o gênero feminino, entre os anos de 2000 a 2009, conforme disposto na Tabela 3.

Tabela 3 - Análise de tendência da série dentre dois períodos considerados para todas as vítimas da região metropolitana.

\begin{tabular}{lcccccc}
\hline Variáveis & \multicolumn{2}{c}{ População geral } & \multicolumn{2}{c}{ Gênero masculino } & \multicolumn{2}{c}{ Gênero feminino } \\
\hline Anos & $\mathbf{2 0 0 0 - \mathbf { 2 0 0 9 }}$ & $\mathbf{2 0 1 0 - \mathbf { 2 0 1 9 }}$ & $\mathbf{2 0 0 0 - \mathbf { 2 0 0 9 }}$ & $\mathbf{2 0 1 0 - \mathbf { 2 0 1 9 }}$ & $\mathbf{2 0 0 0 - \mathbf { 2 0 0 9 }}$ & $\mathbf{2 0 1 0 - \mathbf { 2 0 1 9 }}$ \\
\hline Parâmetros & & & & & & \\
\hline Estatística Z & 1,78 & 1,78 & 1,60 & $-0,53$ & 2,14 & 0 \\
Tamanho da série & 10 & 10 & 10 & 10 & 10 & 10 \\
Tendência crescente* & $0,03^{* *}$ & 0,42 & 0,05 & 0,70 & $0,01^{*}$ & 0,5 \\
Tendência & 0,96 & 0,57 & 0,94 & 0,29 & 0,98 & 0,5 \\
decrescente & & & & &
\end{tabular}

Legenda: ${ }^{*} p$-valor do teste de Mann-Kendall. ${ }^{*}$ Significativo ao nível de $5 \%$.

Fonte: Silva AV, et al., 2021.

A análise espacial dos casos e das taxas médias de suicídio por localidade de residência pode ser observada na Figura 2 abaixo, sendo visualizado uma concentração na região da capital para o número de casos totais, enquanto que o mapa das taxas médias aponta o município de Barra de Santo Antônio como um valor superior as demais áreas da região metropolitana. 
Figura 2 - Distribuição espacial dos casos e das taxas médias de suicídio na região metropolitana de Maceió, 2000-2019.
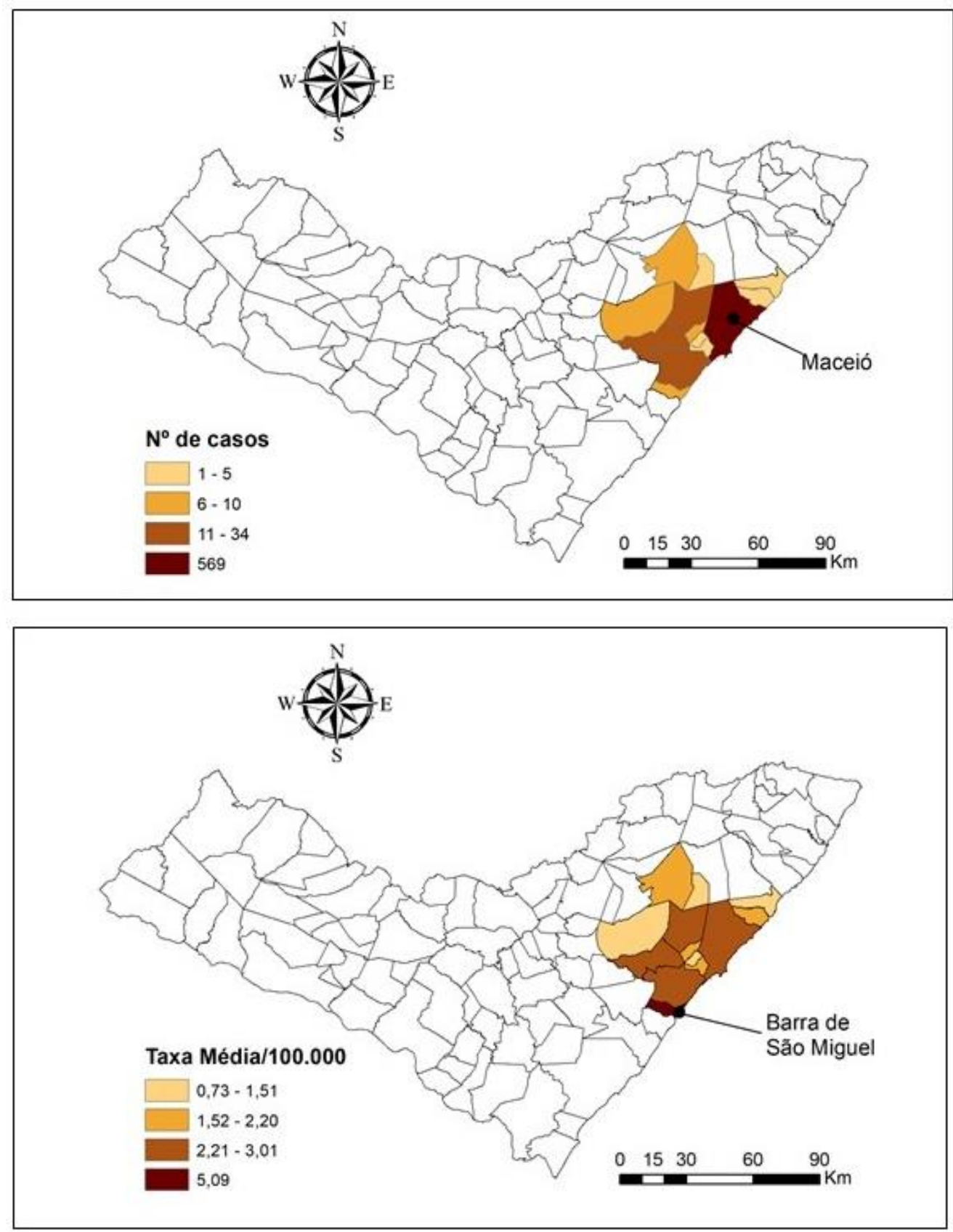

Fonte: Silva AV, et al., 2021; dados extraídos do DATASUS.

\section{DISCUSSÃO}

O presente estudo detalhou a evolução temporal dos casos de suicídio na região metropolitana de Maceió, uma área composta por 13 municípios. Foram descritas as curvas de suicídio ao longo dos anos de acordo com o gênero da vítima, uma vez que houveram diferenças marcantes entre o suicídio masculino e feminino. Além disso, também foram confeccionados os mapas representando o número total de casos e as taxas médias por município. Os dados aqui apresentados são relativos a informações computadas anualmente para cada município da região metropolitana e representam dados agregados, neste sentido trata-se de uma avaliação ecológica do suicídio.

Do total de registros notificados pelo Sistema de Informação de Mortalidade do Ministério da Saúde para os municípios que compõem a região metropolitana de Maceió, foi identificado que cerca de 7 entre cada 
pessoa que cometem o ato suicida são homens, e que a taxa média de suicídio foi 3,3 vezes maior nos homens. Esse padrão masculino de suicídio tem sido confirmado na literatura científica, dados globais extraídos da Organização Mundial de Saúde mostram que os homens cometem três vezes mais suicídio que as mulheres, em especial nos países de alta renda, enquanto que as mulheres lideram as taxas de tentativa de suicídio (WHO, 2014). Essa é uma tendência histórica, já percebida no século XIX por Durkheim, em seus estudos pioneiros sobre o suicídio (DURKHEIN É, 2013).

O fato dos homens cometerem mais suicídio tem sido associado a algumas questões de gênero e do papel masculino no lidar com questões de sentimentos depressivos e suicidas, em geral, o homem está mais associado a maiores níveis de força e agressividade, independência, comportamentos de risco, enquanto que a mulher lida melhor com a solidão e o isolamento social, além de possuírem redes sociais de proteção e engajamento para buscar ajuda para os problemas mentais que enfrentam (ASSOCIAÇÃO BRASILEIRA DE PSIQUIATRIA, 2014; NOCK MK, et al., 2008; STACK S, 2000). Estudo epidemiológico realizado no Brasil sobre o perfil do suicídio entre os anos de 2000 e 2012 identificou que a taxa de suicídio entre os homens foi três vezes maior em relação às mulheres em todas as regiões do país (MACHADO DB e SANTOS DN, 2015).

Dentre os municípios da região metropolitana, a capital apresentou o maior número ao longo de toda a série. Isto é compreensível pelo tamanho populacional que Maceió possui. Segundo o IBGE é estimado que Maceió possui uma população de 1.025.360 habitantes para o ano de 2010, ao passo que todos os demais municípios da região não ultrapassam 100.000. Neste sentido a determinação das taxas por município foi importante para corrigir a discrepância do tamanho populacional. Ao realizar tal procedimento foi verificado que um pequeno município com população estimada de 8.378 habitantes em 2020, denominado de Barra de São Miguel, apresentou a maior taxa média, seguida pela capital Maceió.

Este resultado acima indica que mesmo municípios com tamanhos populacionais tão discrepantes apresentaram as maiores taxas da região metropolitana. Obviamente este resultado precisa ser ponderado, uma vez que as taxas sofrem influência do tamanho populacional, de modo que a ocorrência de um único caso em um município pequeno como Barra de São Miguel influencia significantemente no incremento da taxa.

Considerando a taxa média para toda a região metropolitana o valor de 2,2 pode ser considerado baixo se compararmos com outros estudos em regiões próximas. Em estudo realizado no estado de Sergipe identificou uma taxa média de 5,44 por 100.000 hab. no ano de 2015 (SANTOS AD, et al., 2015). Outro estudo temporal realizado no Estado de Recife encontrou uma taxa média de 4,7 suicídios por 100.000 hab. (BERINGUEL BM, et a., 2020). O coeficiente de mortalidade por suicídio do presente estudo também esteve abaixo do encontrado para a média nacional entre anos 2000 e 2012, cujo coeficiente foi de 5,7 por 100.000 hab. Entretanto ao observar a taxa média para o gênero masculino no atual estudo, percebemos que ela esteve bem próxima da nacional, indicando que para a região metropolitana de Maceió, o gênero masculino é responsável por grande parcela das taxas de suicídio.

Ao observar a evolução nas taxas de suicídio geral da população, envolvendo homens e mulheres, foi estimado um aumento significativo entre o período de 2000-2009, mas no segundo período manteve-se estacionária. Esse aumento do primeiro período foi explicado principalmente pelo aumento resultante de vítimas mulheres, uma vez que o resultado do teste de significância indicou haver aumento significativo apenas para mulheres. Este dado é interessante pois mostra que apesar das taxas serem maiores nos homens, tem havido um aumento significativo nas taxas para as mulheres.

Levando em conta que as mulheres, em geral, apresentam mais comportamentos suicida e tentativas de suicídio, é preocupante esse aumento, ainda que baixo em termos numéricos. Em Alagoas, no período de 2009 a 2018, um estudo realizado incluindo mulheres em idade fértil evidenciou que o número de tentativas de suicídio triplicou no estado em uma década para este grupo populacional (TEIXEIRA LM, et al., 2020). Estudo realizado no Distrito Federal mostrou que das 760 tentativas de suicídios entre os anos de 2015 e 2016, 520 foram apenas entre mulheres (BAÉRE F e ZANELLO V, 2018).

Curiosamente vários municípios da região metropolitana de Maceió apresentaram sucessivas taxas nulas de suicídio em vários anos. Há de se questionar se tais dados refletem a realidade epidemiológica ou se há 
algum tipo de falha no processamento de dados ou subnotificação do fenômeno. Embora seja difícil quantificar e estimar o percentual de subnotificação, algumas explicações têm sido referenciadas como o estigma associado ao suicídio, dificuldades de identificar o evento e falhas ao atestar a causa correta do óbito (MINISTÉRIO DA SAÚDE, 2019; SANTOS AS, et al., 2014; SOARES FILHO AM et al, 2016).

O presente estudo trouxe uma série temporal até o ano de 2019, uma vez que os dados para 2020 ainda não estavam consolidados. Vale ressaltar que o ano de 2020 foi marcado pela pandemia do novo coronavírus, causando a síndrome respiratória SARS-COV-2 pelo novo coronavírus, conhecida por COVID-19, afetando mais de 200 países. Há indícios que o risco de suicídio pode ter aumentado devido ao estigma em relação a indivíduos com COVID-19 e suas famílias, em especial àqueles com transtornos psiquiátricos, haja vista que as medidas adotadas para a prevenção da disseminação do vírus incluíram 0 isolamento social e distanciamento físico entre as pessoas, desencadeando a piora dos sintomas e novos problemas de saúde mental como depressão, ansiedade e estresse pós-traumático - todos associados ao aumento do risco de suicídio (GUNNELL D, et al., 2020).

Importante destacar que a existência prévia de algum transtorno mental, como os transtornos psicóticos, transtornos de humor, ansiedade, uso de substâncias psicoativas, entre outros, eleva significativamente o risco de suicídio (BORBA LO, et al, 2020). Especificamente com relação a depressão estima-se que seja o diagnóstico presente em pelo menos $50 \%$ dos suicídios consumados. Este dado, torna-se preocupante, pois a depressão vem sendo considerada a principal causa de incapacidade em todo o mundo e contribui de forma significativa para a carga global de doenças (WHO, 2017).

Atualmente as mídias sociais e o uso da internet têm um papel primordial quando atuam em estratégias de prevenção ao suicídio. Alguns websites que promovem e divulgam informações sobre a saúde mental já estão mostrando resultados positivos na busca de ajuda pelos usuários. Nesse contexto, podem ser citados como bons exemplos os chats on-line com profissionais treinados para ouvir pessoas suicidas, além de programas de autoajuda e terapia on-line, porém mesmo com o aumento da pesquisa e conhecimento sobre o tema, o tabu e o estigma em torno do suicídio persiste e muitas vezes faz com que as pessoas não procurem ajuda, portanto, a conscientização da comunidade e a quebra dos tabus são importantes atitudes para a prevenção do suicídio (WHO, 2014).

Em contrapartida, a facilidade de acesso as chamadas redes sociais disponíveis na internet, tais como o Facebook, Instagram, WhatsApp, Telegram e Snapchat, acabou se tornando um facilitador para uma gama de suicídios em massa, tal efeito denomina-se efeito Werther (pico de conexões de suicídios depois de um suicídio amplamente divulgado). Ao contrário deste, existe o efeito Papageno, quando uma superação é amplamente divulgada servindo de inspiração a outras pessoas a desistirem de algum ato que coloque sua vida em risco, como é o exemplo da campanha mundial de prevenção ao suicídio, o "setembro amarelo" (BLATT MR, 2019).

A principal limitação do presente estudo diz respeito a impossibilidade de inclusão do ano de 2020 dentro da análise devido ao fato de que tais dados ainda não estavam consolidados e disponibilizados pelo Ministério da Saúde no momento de finalização da presente pesquisa. Além deste fator, temos a questão da subnotificação do suicídio que é inerente ao tipo de fenômeno em análise. Tais limitações não foram impeditivas para realização de inferências válidas, uma vez que utilizamos os dados oficiais de registro do suicídio disponibilizados pelo governo.

\section{CONCLUSÃO}

A violência suicida na região metropolitana indicou uma baixa taxa ao longo de 20 anos, estando os homens mais frequentemente associados às taxas mais altas em relação às mulheres. Apesar disso, apenas no gênero feminino é que foi verificado um aumento significativo entre os anos de 2000 a 2009. A capital concentrou o maior número absoluto de casos, enquanto que o município de Barra de São Miguel exibiu a maior taxa média. O presente trabalho contribui para uma maior compreensão da epidemiologia do suicídio para a região metropolitana de Maceió, servindo de base para análises mais aprofundadas acerca do fenômeno nesta área. Análises futuras podem investigar a problemática em regiões do agreste e do sertão para verificação de possíveis semelhanças ou divergência da região em análise. 


\section{REFERÊNCIAS}

1. ASSOCIAÇÃO BRASILEIRA DE PSIQUIATRIA (ABP). Suicídio: informando para prevenir. Brasil, 2014. Disponível em: https://repositorio.observatoriodocuidado.org/handle/handle/2522. Acesso em: 21 de agosto de 2021.

2. ALMEIDA-FILHO N, BARRETO ML. Epidemiologia e Saúde: fundamentos, métodos e aplicações. Rio de Janeiro: Guanabara Koogan, 2011; $175 p$.

3. BAÉRE F, ZANELLO V. O gênero no comportamento suicida: Uma leitura epidemiológica dos dados do Distrito Federal. Estudos de Psicologia, 2018; 23(2): 168-78.

4. BERINGEL BM, et al. Mortalidade por suicídio no Estado de Pernambuco, Brasil (1996-2015). Revista Brasileira de Enfermagem, 2020; 73(Suppl 1): e20180270.

5. BLATT MR. A relevância das redes sociais na prevenção ao suicídio. Revista da Saúde da AJES, 2019, 5(10): 36-46.

6. BORBA LO, et al. Fatores associados à tentativa de suicídio por pessoas com transtorno mental. Revista Mineira de Enfermagem, 2020, 24: e1284.

7. BOTEGA NJ. Crise suicida. São Paulo: Artmed, 2015.

8. BRASIL. Decreto-Lei № 3.689, de 3 de outubro de 1941. Código de Processo Penal. Disponível em: http://www.planalto.gov.br/ccivil_03/decreto-lei/del3689.htm. Brasil, 1941. Acesso em 21 de agosto de 2021.

9. DURKHEIN, É. O suicídio: Estudo de sociologia. São Paulo: Edipro, 2013.

10. GUNNELL D, et al. Suicide risk and prevention during the COVID-19 pandemic. The Lancet Psychiatric, 2020; 7(6): 468-71.

11. INTERNATIONAL CLASSIFICATION OF DISEASE AND RELATED HEALTH PROBLEMS (IDC). Chapter XX. $10^{\text {th }}$ revision. Version 2016. Disponível em: https://icd.who.int/browse10/2016/en. Acesso em: 21 de agosto de 2021.

12. MACHADO DB, SANTOS DN. Suicídio no brasil, de 2000 a 2012. Jornal Brasileiro de Psiquiatria, 2015; 64(1): 45-54.

13. MAGNANI RM, STAUDT AC. Estilos parentais e suicídio na adolescência: uma reflexão acerca dos fatores de proteção. Pensando famílias, 2018; 22(1): 75-86.

14. MINISTÉRIO DA SAÚDE. Agenda de ações estratégicas para a vigilância e prevenção do suicídio e promoção da saúde no Brasil: 2017 a 2020. BRASIL, 2020. Disponível em: http://bvsms.saude.gov.br/bvs/saudelegis/gm/2009/prt2008_01_09_2009.html. Acesso em: 21 de agosto de 2021.

15. MINISTÉRIO DA SAÚDE. Departamento de Informática do SUS (DATASUS). Informações em Saúde. Demográficas e Socioeconômicas. População Residente. Estudo de estimativas populacionais por municípo, idade e sexo - 20002020. Brasil, 2020. Disponível em: http://tabnet.datasus.gov.br/cgi/deftohtm.exe?popsvs/cnv/popbr.def. Acesso em: 21 de agosto de 2021.

16. MINISTÉRIO DA SAÚDE. Departamento de Informática do SUS (DATASUS). Informações em Saúde. Estatísticas Vitais. Mortalidade - 1996 a 2019, pela CID-10. Brasil, 2020. Disponível em: http://www2.datasus.gov.br/DATASUS/index.php?area=0205. Acesso em: 21 de agosto de 2021.

17. MINISTÉRIO DA SAÚDE. Secretaria de Vigilância em Saúde. Boletim epidemiológico 15. Suicídio: tentativas e óbitos por intoxicação exógena no Brasil, 2007 a 2016, 2019; 50: 1-12.

18. NOCK M, et al. Suicide and suicide behavior. Epidemiologia e Serviços de Saúde, 2008; 30(1): 133-54.

19. SANTOS AD, et al. Spatial analysis and temporal trends of suicide mortality in Sergipe, Brazil, 2000-2015. Trends in Psychiatry and Psycotherapy, 2018; 40(4): 269-76.

20. SANTOS SA, et al. Tentativas e suicídios por intoxicação exógena no Rio de Janeiro, Brasil: análise das informações através do linkage probabilístico. Cadernos de Saúde Pública, 2014; 30(5): 1057-66.

21. SILVA DA, MARCOLAN JF. O impacto das relações familiares no comportamento suicida. Research, Society and Development, 2021; 10(2): e17310212349.

22. SOARES FILHO AM et al. Revisão dos métodos de correção de óbitos e dimensões de qualidade da causa básica por acidentes e violências no Brasil. Ciência e Saúde Coletiva, 2016; 21(12): 3083-18.

23. STACK S. Suicide: a 15-year review of the sociological literature part I: cultural and economic factors. Suicide and LifeThreatening Behaviour, 2000; 30(2):145-62.

24. TEIXEIRA LM, et al. Mortalidade por suicídio em mulheres com idade fértil. Research Society and Development, 2020; 9(11): e419119565.

25. WORLD HEALTH ORGANIZATION (WHO). Preventing suicide: a global imperative. 2014. Dispnível em: https://www.who.int/publications/i/item/9789241564779. Acesso em: 21 de agosto de 2021.

26. WORLD HEALTH ORGANIZATION (WHO). Depression and other common mental disorders: global health estimatres. DIsponível em: https://apps.who.int/iris/bitstream/handle/10665/254610/WHO-MSD-MER-2017.2-eng.pdf. Acesso em 25 de agosto de 2021. 